\title{
Multiphysic Simulations Approach toward Better Functional Modeling of An EM Transduction Pressure Sensor
}

\author{
Mingli Ding, Xiaobing Li \\ Dept. of Automatic Test and Control, Harbin Institute of Technology, Harbin 150001, China
}

Received: 1 Oct. 2012, Revised: 22 Dec. 2012, Accepted: 25 Dec. 2012

Published online: 1 Jun. 2013

\begin{abstract}
Based on the electromagnetic transduction principle, a passive and wireless pressure sensor with a high sensitivity is designed. In the passive and wireless pressure sensor, the applied pressure generates a displacement of a thin membrane located above a planar millimeter-wave resonator. However, in previous works, the real membrane deflection has not been incorporated in the electromagnetic simulations and a uniform membrane deflection was assumed instead. In order to have a functional estimation of the real measurement unit, multiphysic simulations have to be performed with consideration of the electromagnetic and mechanical properties of the sensor. Therefore, this paper presents a progressive approach to derive an accurate functional approximation of the sensor under the real working condition and to take into account the real deflection of the membrane when applying a pressure.
\end{abstract}

Keywords: Pressure sensor, electromagnetic transduction principle, multiphysic simulations

\section{Introduction}

Pressure sensors play an important role in the fields of environment monitoring, automotive, biomedical and industrial applications [1-3]. Nowadays the development of wireless technology and silicon micromachining allows the realization of wireless micro-systems for remote sensing applications [4-5].

In previous works, a wireless pressure sensor based on the electromagnetic (EM) transduction has been proposed, analyzed and designed [6-8]. Such wireless EM pressure sensor is composed of four parts: a substrate, a coplanar resonator, an air cavity and a membrane. The 3D view of the sensor structure is shown in Fig. 1.1. The resonator is placed in the cavity which is sealed to a high resistivity silicon membrane. If a pressure is applied on the membrane surface, a deflection of the membrane can be observed. Such deflection causes the modification of the electromagnetic field distribution around the resonator, and a shift in the resonant frequency also occurs [9]. From the measurement of this frequency shift, the applied pressure can be derived. In previous work, electromagnetic simulations were presented with assuming a uniform membrane displacement. This approximation makes the design of the pressure sensor easier and more rapid, but it does not reflect the real working status of the sensor. Furthermore, based on this approximation, pressure cells with a $50 \mu \mathrm{m}$ high resistivity silicon (HRSi) membrane were fabricated and a sensitivity of $370 \mathrm{MHz} / \mathrm{bar}$ was measured.

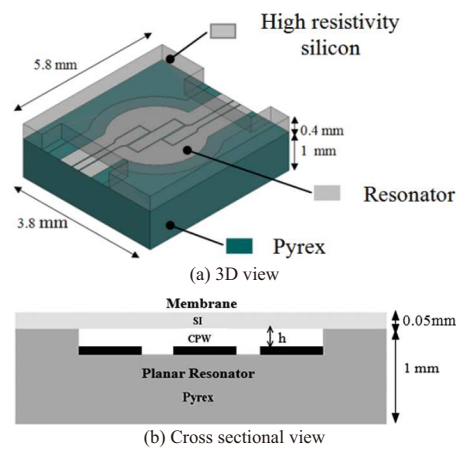

Fig. 1 Wireless pressure sensor structure

\footnotetext{
*Corresponding author e-mail: dingml@ hit.edu.cn
} 
For a better and more accurate understanding of the operating mode of the multiphysic (mechanical and electromagnetic) passive sensor, this paper incorporates the real deflection of the membrane in the simulation. Preliminary results of this work have been reported in [10]. The impact of the sensors characteristic parameters-such as the membrane shape, the membrane deflection and the membrane thickness - on performance of the pressure measurement cell is also presented and discussed.

\section{EM Simulation Results with a Cylindrical Membrane Profile}

In previous design, the sensor HRSi membrane is modeled as a rectangular box. In this section, another membrane shape is investigated. As shown in Fig. 2.1, a cylindrical shape is chosen to replace the box. By changing the geometrical parameters of the cylinder, one can monitor the effect of the membrane shape on the sensor functioning, and the change of the cylinder position can be used to indicate the thickness of air gap 'h'.

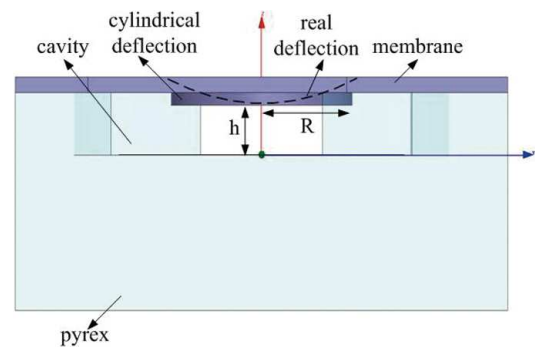

Fig. 2 Cross sectional view of the sensor structure with cylindrical membrane shape (dashed line indicates the real membrane deflection)

EM simulations are performed by using Ansoft HFSS (High Frequency Structure Simulator). This commercial software is a 3D volumetric Full-Wave field solver in frequency domain based on the finite elements method (FEM). The resonant frequency of the structure versus the air gap thickness ' $h$ ' with different cylinder radius ' $R$ ' is shown in Fig. 2.2.

It can be observed in Fig. 2.2, by increasing the cylinder radius, the frequency value drops at the same thickness of air gap. The air gap is between the cylinder and the CPW, $r_{1}$ and $r_{2}$ are the radius of the two cylindrical membranes respectively $\left(r_{2} \dot{i} r_{1}\right)$, shown in Fig. 2.3. Assume that the maximal air gap thickness is $6 \mu \mathrm{m}$, and a certain pressure is applied on the surface of the cylindrical membrane, which produces the same deflection $(\mathrm{h}=2 \mu \mathrm{m})$. If there is no deflection, the

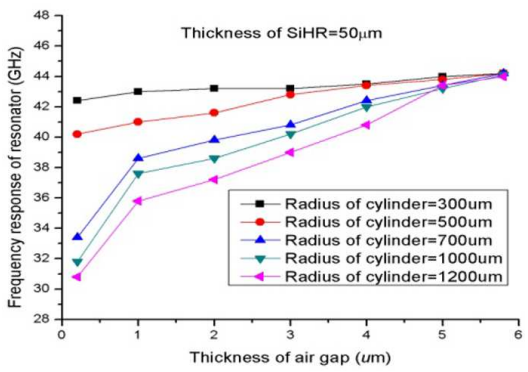

Fig. 3 Sensor resonant frequency with the cylindrical membrane shape versus air gap thickness

effective permittivity of the air cavity $\left(\xi_{r}\right)$ is unaffected and $\xi_{r}=1$. If there is a deflection caused by the pressure, $\xi_{r}$ will not be a constant and increases. Under the same deflection, from Fig. 2.3, the cylinder of $r_{2}$ has larger interaction with the resonator than that of $\mathrm{r}_{1}$, then the $\xi_{r}\left(\mathrm{r}_{2}\right)$ is larger than $\xi_{r}\left(\mathrm{r}_{1}\right)$. The frequency response of the structure is conversely proportional to $\xi_{r}$. So that is why under the same deflection, the cylindrical structure with larger radius has smaller frequency response.

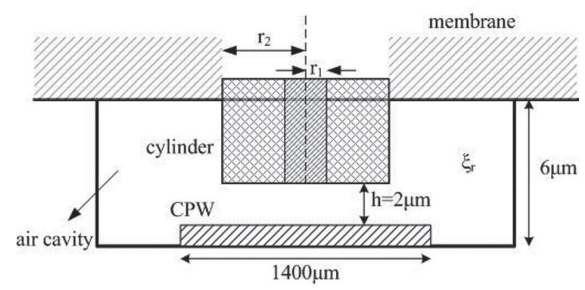

Fig. 4 Illustration of the structure with cylindrical membrane shape having different radius values

\section{EM Simulation Results with real Membrane Deflection}

Based on the sensor structure, the shape and the thickness of the silicone membrane, a certain range of pressure can be defined. The deflection $W(r)$ of the membrane can be established as follows

$$
W(r)=W_{0} \cdot W_{N}(r),
$$

where $W_{0}$ is the maximum deflection in the center of the membrane; $W_{N}(r)$ is a pressure independent function. For a circular membrane, if the radius is dented identified by $R$ and the uniform thickness ise, $W_{0}$ and $W_{N}(r)$ can be written as follows

$$
W_{0}=\frac{12 R^{4}\left(1-v^{2}\right)}{64 E e^{3}} P ; \quad W_{N}(r)=\left(1-\frac{r^{2}}{R^{2}}\right)^{2},
$$


where $P$ is the pressure, $E$ is the Young modulus and $v$ is Poisson's ratio. Here, $E=168.9 \mathrm{GPa}, v=0.0625, e=50 \mu$ $\mathrm{m}, R=1400 \mu \mathrm{m}$ and $r=1000 \mu \mathrm{m}$.

Fig. 3.1 shows the real membrane deflection given by Eq.(1)-(2). Fig. 3.2 depicts the deflection model done on HFSS in the former work.

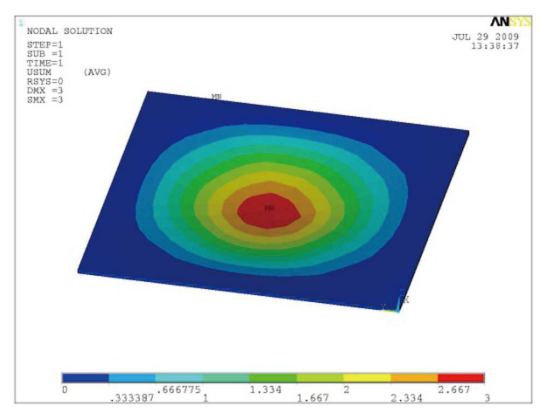

Fig. 5 Real membrane deflection realized by ANSYS.

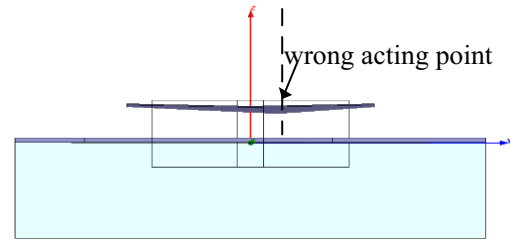

Fig. 6 Wrong membrane deflection.

In the electromagnetic simulation, a modeling difficulty is observed. It can be seen in Fig.3.2 that, when a pressure is acted on the membrane, the acting point is not in the center of the membrane. This kind of deflection does not match the actual membrane deflection. Some difficulties were also observed when importing the membrane profile, obtained on mechanical simulator, on the EM simulator. In this section, a method is proposed to solve this problem: the membrane is split into four parts, and then, each quarter of the membrane is used to reform the whole membrane as shown in Fig. 3.3.

After getting the correct membrane deflection profile, the pressure sensor cell simulation is done. For the simulations, the membrane thickness is assumed to be equal to $50 \mu \mathrm{m}$. The air gap thickness ' $\mathrm{h}$ ' is ranged from 0 to $60 \mu \mathrm{m}$. Fig. 3.4 shows the simulation results.

Fig. 3.4 shows the frequency response in case of uniform membrane displacement and real membrane deflection. Comparing them, it can be seen that the resonant frequency value becomes larger after splitting the membrane. The curves tendencies are similar: by increasing the air gap thickness, the frequency tends to increase. One can notice that by increasing the air gap thickness, the resonant frequencies in the two situations converge to the same 4 value (around $55 \mathrm{GHz}$ ). That is because for big air gap thickness value, the membrane is

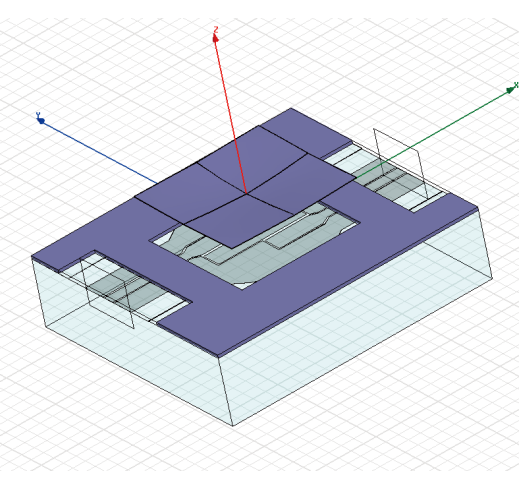

Fig. 7 Split membrane profile.

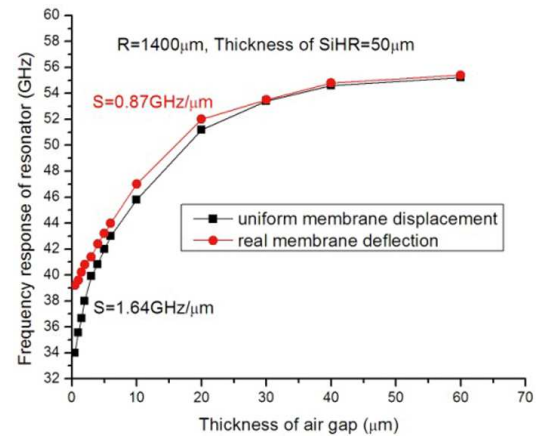

Fig. 8 Frequency responses versus air gap thickness: Comparison between uniform membrane displacement and real membrane deflection.

far from the resonator. From the sensitivity point of view, it is obvious that the former structure with uniform membrane deflection has larger sensitivity. This is due to the fact that in this condition, the EM field surrounding the coplanar resonator has larger interaction area with the High Resistivity silicon membrane than the structure of with real membrane deflection for an equivalent applied pressure.

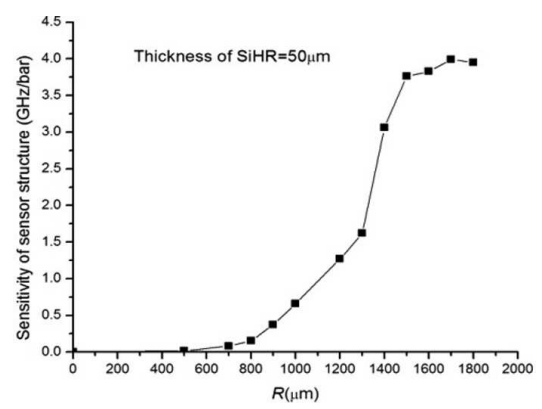

Fig. 9 Sensor sensitivity versus the membrane radius. 
Another parameter to take into account: the circular membrane radius 'R'. Fig. 3.5 exhibits the sensor sensitivity versus the membrane radius. Due to the structure size, the largest radius ' $R$ ' which can be modeled is about $1800 \mu \mathrm{m}$.

From Fig. 3.5, it can be seen that by increasing the radius ' $R$ ', the sensitivity tends to increase. When $\mathrm{R}=900 \mu \mathrm{m}$, the sensitivity is $0.37 \mathrm{GHz} / \mathrm{bar}$. This sensitivity value is very close to the measured one6. In the experiment, the radius of the pressure probe module (PPM) nozzle, used to apply pressure, is $700 \mu \mathrm{m}$. In the experiment, the membrane is not sealed and that's why when using a PPM nozzle of $700 \mu \mathrm{m}$, it seems that we have an applied pressure on a $900 \mu \mathrm{m}$ membrane radius.

Fig. 3.6 presents the resonant frequency versus the air gap variation for different membrane thickness values.

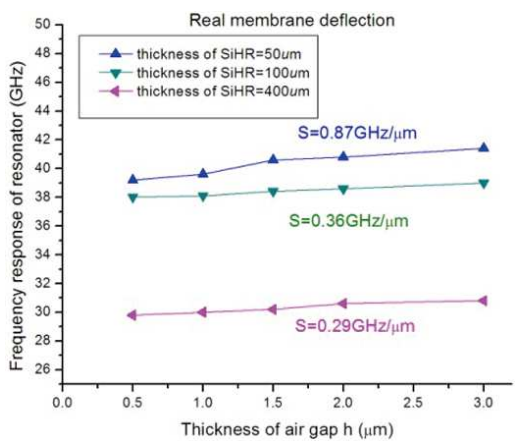
(a) Real membrane deflection

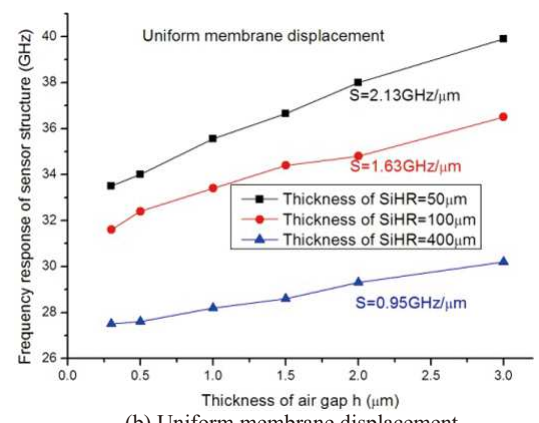

(b) Uniform membrane displacement

Fig. 10 Simulated sensor sensitivities for real membrane deflection and uniform membrane displacement.

From Fig. 3.6, one can notice that by increasing the membrane thickness, a decrease of the resonant frequency is obtained for the same air gap value. By increasing the membrane thickness, the sensitivity tends to drop. In the case of real membrane deflection, the sensitivity is slightly smaller than that of the uniform membrane.

Fig. 3.7 presents the simulated sensitivities, under real membrane deflection, for two pressure cell with different radius ' $R$ ' ( $(1400 \mu \mathrm{m}$ and $900 \mu \mathrm{m})$.

When ' $\mathrm{R}$ ' $=1400 \mu \mathrm{m}$, the curve displays a peak value (Sensitivity $=4.25 \mathrm{GHz} / \mathrm{bar}$ ) for a membrane thickness of

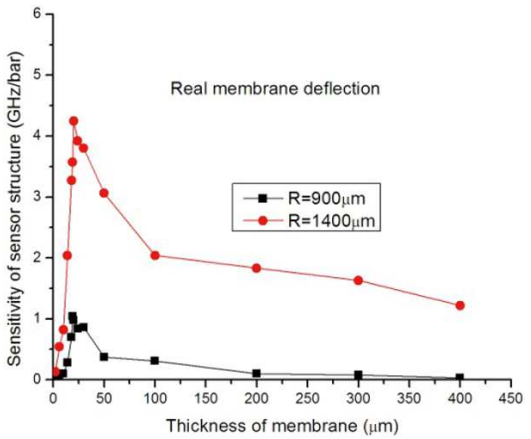

Fig. 11 Sensitivity curve versus the membrane thickness.

$20 \mu \mathrm{m}$. When the membrane thickness increases, the sensitivity is reduced significantly. By decreasing the thickness values from $20 \mu \mathrm{m}$, the sensitivity also tends to decrease. Obviously, at the membrane thickness of $20 \mu$ $\mathrm{m}$, which is the best choice for the membrane, the sensor has the maximal sensitivity.

\section{EM Simulation Results with Real Membrane Deflection and Metalized HRSI}

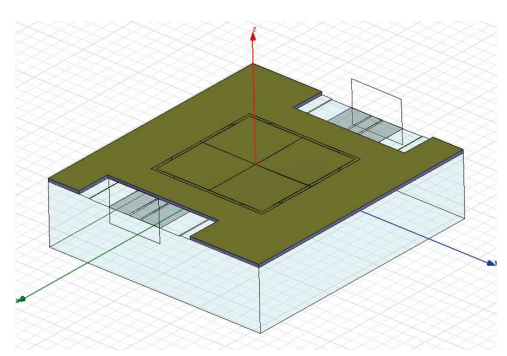

Fig. 12 Sensor structure with PEC (real membrane deflection)

PEC (Perfectly Electric Conducting) materials are those that exhibit infinite conductivity and do not produce losses. Comparing to real material, PEC is an idealized material and it is much more suitable for simulating than real material with finite conductivity. We hope to through the simulation, find out the sensitivity change comparing with the structure without PEC. In this simulation work, the two deflections (uniform membrane displacement and real membrane deflection) are considered respectively. Fig. 4.1 shows the sensor structure with PEC (on the top), which has the thickness of $10 \mu \mathrm{m}$ and covers the membrane.

Fig. 4.2 depicts the frequency response of the sensor structure with PEC under the uniform membrane displacement and the real membrane deflection 


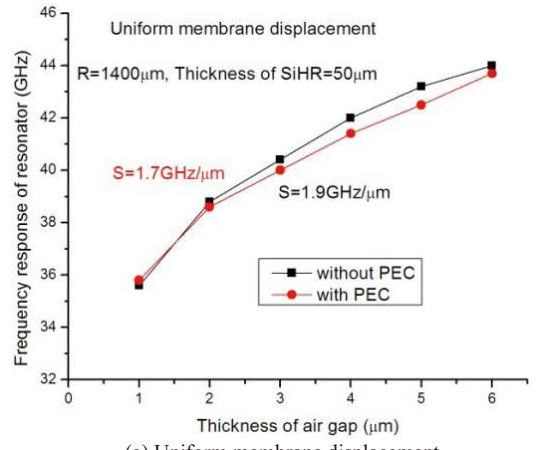

(a) Uniform membrane displacement

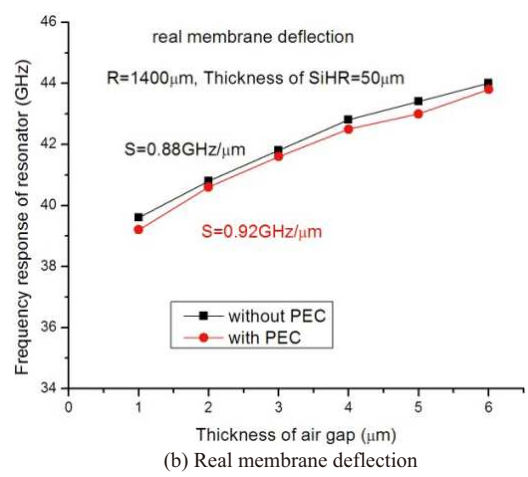

Fig. 13 Frequency response of the sensor structure with PEC

respectively. Looking to Fig. 14(a), it can be found that, with PEC, at the same thickness of air gap, the frequency value is a slightly smaller than that without PEC. The phenomenon is due to PEC which makes the effective permittivity of the media larger, so the frequency response of the structure drops. Comparing the sensitivity, the structure with PEC has a close sensitivity to that without PEC. A slightly larger sensitivity $(\mathrm{S}=1.9 \mathrm{GHz} / \mu$ $\mathrm{m}) \mathrm{can}$ be acquired under the structure with PEC. The similar results can be acquired in Fig. 14(b), which is based on the structure of real membrane deflection without PEC. The simulation results indicate that the sensor structure with PEC can decrease the frequency response and make a slight enhancement to the sensitivity for measuring the pressure.

\section{Conclusions}

In this paper, a functional estimation based on multiphysic simulations approach is presented. Because of its structural complexity, a progressive simulation approach is applied to take into account the electromagnetic and the mechanical properties of the sensor. A comparison between the simulation and the measurement results becomes more relevant than before. From measurement point of view, a sensitivity of 370 $\mathrm{MHz} / \mathrm{bar}$ is observed for a $50 \mu \mathrm{m}$ HRSi circular membrane. The fabricated membrane radius is $1400 \mu \mathrm{m}$ and the dimensions of the PPM's nozzle used for applying pressure are $700 \mu \mathrm{m} \times 700 \mu \mathrm{m}$. From simulation point of view, the real membrane deflection is modeled on HFSS. Based on the sensor geometric parameters, investigations were performed and permitted a better functional explanation of the EM pressure sensor. Good agreement with measurement is obtained for a simulated radius 'R' $=900 \mu \mathrm{m}$.

\section{Acknowledgements}

This work is partially supported by the National Natural Science Foundation of China (Grant No. 60901042 and No. 61171196). Thanks for the help.

\section{References}

[1] Boca Raton, The MEMS handbook, FL CRC Press. (2002).

[2] Lee D. and Won D., A Study on Security Management Service System for Wireless Network Environment, Applied Mathematics \& Information Sciences, 6 (2012) 209-220.

[3] D. S. Ballantine, R. M. White, S. J. Martin, A. J. Ricco, G. C. Frye, E. T. Zellers, and H. Wohltjen, Acoustic Wave Sensors: Theory, Design and Physicochmical Applications, Boston, MA: Academic Press. (1997).

[4] H. E. Endres, R. Hatinger, M. Schwaiger, G. Gmelch, and M. Roth. A capacitive $\mathrm{CO}_{2}$ sensor system with suppression of the humidity interference, Sensors and Actuators B, 57 (1999) 83-87.

[5] Zuo X., Gao W., and Zhang G., Design of Environmental Parameters Monitoring System for Watermelon Seedlings Based on Wireless Sensor Networks, Applied Mathematics \& Information Sciences, 5 (2011) 243-250.

[6] M. M. Jatlaoui, F. Chebila, P. Pons, H. Aubert, Pressure Sensing Approach Based on Electromagnetic Transduction, 2008 IEEE Asia Pacific Microwave Conference, Hong Kong, (2008) 16-19.

[7] M. M. Jatlaoui, P. Pons, H. Aubert, Radio Frequency Pressure Transducer, $200737^{\text {th }}$ European Microwave Conference, Munich, (2007) 736-739.

[8] M. M. Jatlaoui, F. Chebila, I.Gmati, P.Pons, H.Aubert. New Electromagnetic Transduction Micro-sensor Concept For Passive Wireless Pressure Monitoring Application, the 15th Conference on Solid-State Sensors, Actuators \& Microsystems, Transducers 2009, Denver, Colorado, USA, (2009) 1742-1745.

[9] Abdel-Wahab N. H., The Interaction Between a Four-Level $N$-Configuration Atom and a Single Mode Cavity Field, Applied Mathematics \& Information Sciences, 1 (2007) 263273.

[10] D. Mingli, M. M. Jatlaoui, P. Pons, H.Aubert, Multiphysic Simulations Approach for an EM Transduction Based Pressure Sensor, the 20th Workshop on Micromachining, micro-mechanics and Micro-systems (MME2009), Toulouse, France, (2009) D20. 


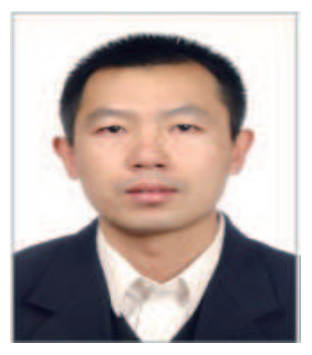

Mingli Ding received M.S., and Ph.D. Degrees in Department of Automatic Test and Control from Harbin Institute of Technology, China, in 2002 and 2005, respectively. He is currently an associate professor with the Department of Automatic Test and Control of Harbin Institute of Technology, China. His research interests include Wireless Sensor Design and Multisource Information Fusion.

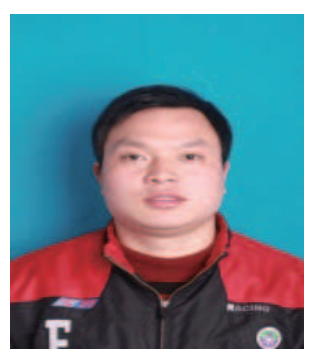

Xiaobing $\mathbf{L i}$ received B.S. Degrees in Department of Automatic Test and Control from NanChang University, China, in 2012. $\mathrm{He}$ is currently a postgraduate student with the Department of Automatic Test and Control of Harbin Institute of Technology, China. His research interests include Wireless Sensor Design. 